ISSN: 1948-9900

(C)2013 Science Publications

doi:10.3844/amjnsp.2013.13.24 Published Online 4 (1) 2013 (http://www.thescipub.com/ajn.toc)

\title{
Stem Cells in Traumatic Brain Injury
}

\author{
${ }^{1}$ Samuel Dobrowolski and ${ }^{1,2}$ Guilherme Lepski \\ ${ }^{1}$ Department of Neurosurgery, University of Tuebingen, Tuebingen, Germany \\ ${ }^{2}$ Department of Neurology, Universidade de São Paulo, São Paulo, Brazil
}

Received 2013-03-19, Revised 2013-04-03; Accepted 2013-04-12

\begin{abstract}
Traumatic Brain Injury (TBI) is a devastating clinical condition that often causes permanent incapacity, especially in the younger population. The clinical relevant of TBI justifies the scientific interest in the pathophysiology of TBI, as well as in protective effects and development of treatment options. Stem cells have the ability to induce neuroprotection and neural repair inflammatory suppression, causing tissue reconstruction completely or partially damaged cells to preventing cell death to evolve. However the neurological improvement observed in preclinical studies and clinical tests based on neurological and behavioral disorders and the mechanism of action of stem cells remains unknown. In this study the authors discuss the current status of using stem cells to treat TBI, including the basic cell types and potential mechanisms of action, preclinical data and points out lack of studies and hurdles for clinical application. The authors also focusing on the recent demonstration that neurogenesis occurs in all mammals throughout adult life, although at a low rate, is possible to induce neurogenesis de novo in the adult mammalian brain, particularly in the neocortex where it does not normally occur and that it may become possible to manipulate endogenous multipotent precursors in situ to replace lost or damaged neurons. Elucidation of the relevant molecular controls may both allow control over transplanted precursor cells and potentially allow the development of neuronal replacement therapies for neurodegenerative disease and other central nervous system injuries that do not require transplantation of exogenous cells. Discuss strategies of enhance the neurogenesis (for example by exogenous tropics factor administration) and the transplantation of different types of neural progenitor cells after TBI. Each strategy is discussed with an emphasis on highlighting the progress and limiting factors relevant to the development of clinical trials of cellular replacement therapy for severe TBI in humans.
\end{abstract}

Keywords: Stem Cells, Traumatic Brain Injury, Adult Neurogenesis, Progenitor Migration

\section{INTRODUCTION}

Traumatic Brain Injury (TBI) is a devastating clinical condition that often causes permanent incapacity, particularly in the younger population. The clinical relevance of TBI justifies the scientific interest in the pathophysiology of TBI, protective effects and development of treatment options.

The extent of the neurologic deficits caused by lesions is determined by two main factors: The primary mechanical insult and the secondary insult caused by inflammation, compression and ischemia (Cheng et al., 2012). The primary lesion is caused by the trauma itself and involves cellular death and tissue necrosis, independently of biological factors. The mechanisms underlying secondary lesions involve activation of inflammation, tissue ischemia, reperfusion deficits, edema, lipidic peroxidation, calcium influx and particularly apoptosis. These secondary lesions constitute the main target for the development of novel therapeutic approaches (Cheng et al., 2012). Among these novel strategies, new neuroprotective agents are being developed, with researchers conducting trials on therapeutic agents such as levodopa/carbidopa as well as several neurotrophic factors. The efficacy of these drugs however, has yet to be confirmed. Other substances Corresponding Author: Samuel Dobrowolski, Department of Neurosurgery, University of Tuebingen, Tuebingen, Germany 
including cannabinoid dexanabinol, erythropoietin and gamaglutamylcysteine ethyl ester have demonstrated neuroprotective effects in humans at an experimental stage, where their administration might limit tissue damage and increase the potential for clinical recovery (Lok et al., 2011).

Stem cells have the ability to induce neuroprotection, inflammatory suppression and neural repair, allowing reconstruction of totally damaged tissues or preventing partially damaged cells from evolving to cell demise (Erceg and Stojkovic, 2009). However, the neurological improvements observed in pre-clinical and clinical trials have been based on results of neurological and behavioral tests, while the underlying mechanism of action of stem cells remains unknown. The percentage of stem cells able to differentiate into neurons is believed to be negligible or even non-existent, with improvements seen in pre-clinical and clinical trials attributable to the secretion of trophic factors triggering endogenous repair mechanisms (Heile and Blinker, 2011; Yang et al., 2012). In addition, tentative pre-clinical data reveals obstacles in the interpretation and translation of the preliminary potential for treatment of TBI in humans.

\subsection{Physiopathology of TBI}

While knowledge on stem cells has advanced in recent years, knowledge on the physiopathology of TBI has lagged, severely hampering the search for an effective stem cell-based therapy in TBI.

\subsection{Primary Lesion}

Primary lesion is caused by impact to the cortical and subcortical brain structures, causing focal or Diffuse Axonal Injury (DAI) and rupture of the Blood Brain Barrier (BBB) (Albert-Weibenberger et al., 2012). The primary event is accompanied by a huge ionic influx known as traumatic depolarization. The main inflammatory neurotransmitters released are the excitatory aminoacids. This may explain the physiopathology of DAI in TBI. Brain edema caused by rupture of the BBB and swelling of astrocytes raises intracranial pressure (Kahle et al., 2013). Vascular endothelial growth factor also plays a role in the rupture of neuronal tissue and increases permeability of the BBB through the synthesis and release of nitric oxide (Woodcock and Morganti-Kossmann, 2013).

\subsection{Secundary Lesion}

Secondary lesion is associated with the release of excitatory aminoacids, oxygen radicals and with the production of nitric oxide, leading to activation of $\mathrm{N}$ Methyl-D-Aspartate (NMDA), 2-amino-3-propanoic acid (5-mthyl-3- oxo-1, 2-oxazol-4-il) (AMPA), alpha-7 nicotinic receptor $(\alpha 7)$, Nicotinic Acetylcholine Receptor (NACR) and calcium influx (Hinzman et al., 2012; Kelso and Oestreich, 2012). This cascade of events promotes mitochondrial rupture and release of free radicals with tissue peroxidation. One theory holds that the release of excitatory aminoacids leads to calcium influx in neurons and other brain cells, promoting oxygen-free radical reactions. The increased calcium and presence of free radical molecules creates an unstable environment in which the cell can increase the production and release of nitric oxide and excitatory aminoacids (e.g., glutamate) (Oliva et al., 2012; Hinzman et al., 2012). Nitric oxide can participate in reactions of oxygen radicals and in lipid peroxidation among neighboring cells (Xiong et al., 2009). Secondary lesions constitute the main target for the development of novel therapeutic approaches (Kumar and Loane, 2012) given they are the main determinant of morbidity and mortality in TBI. To date, several genes have been implicated for influencing outcome after TBI with APOE being the most extensively studied. The proposed mechanism by which Apolipoprotein E (APOE) affects the clinic pathological consequences of TBI is multifactorial and includes amyloid deposition, disruption of cytoskeleton stability, cholinergic dysfunction, oxidative stress, neuroprotection and central nervous system plasticity in response to injury. The Catechol-O-Methyltransferase (COMT) and Dopamine Receptor D2 (DRD2) genes have been less extensively studied and may influence dopamine dependent cognitive processes such as executive/frontal lobe functions. Inflammation, a prominent component in the path physiological cascade initiated by TBI, is in part mediated by the interleukin genes, while the apoptosis that occurs as a consequence of TBI may be modulated by polymorphisms of the p53 gene. The Angiotensin Converting Enzyme (ACE) gene may affect TBI outcome via mechanisms of cerebral blood flow and/or auto regulation whereas the calcium channel, voltage-dependent, P/Q type, alpha 1A subunit (CACNA1A) gene may exert an influence via the calcium channel and its effect on delayed cerebral edema. Although several potential genes that may influence outcome after TBI have been identified, future investigations are needed to validate these genetic studies and identify new genes that might influence outcome following TBI (Dardiotis et al., 2012).

\subsection{Therapeutic Application of Stem Cells in TBI}

For therapeutic application, separate stem cells can be divided into adult or embryonic types, exogenous 
or endogenous origin and local or systemic routes of administration.

\subsection{Exogenous Embryonic Stem Cells (ESCs)}

Embryonic Stem Cells are pluripotent cells capable of differentiating into any of the cell types. ESCs offer the advantage of allowing a large number of cells to be expanded in culture and can produce specific neuron types for transplantation (Leao et al., 2010). This characteristic is relevant in that stem cell replacement therapies require a large number of cells. However, ESCs have the drawback of ethical and religious issues, are prone to rejection upon transplantation and can create teratomas when administered in vivo (Qu et al., 2012).

Studies of transplantation of ESCs in animal models have shown good results. However, broader knowledge is needed before running these studies in humans (Abdullah et al., 2012).

Classically, ESCs have been the most common source of Neural Stem Cells (NSCs), although recent studies have isolated NSCs from areas with active neurogenesis in the Subventricular Zona (SVZ) of the lateral ventricle of rats (Foti et al., 2013).

\subsection{Exogenous Adult Neural Stem Cells (NSCs)}

NSCs are multipotent cells, i.e., have limited potential for differentiation into cells of other tissue types. NSCs can be identified by the immunocytochemical markers nestin, an intermediate filament (Cristini et al., 2011), musashi 1, an RNAbinding protein (Reis and Hermanson, 2012) and the transcription factors Sox1 and Sox2 (Archer et al., 2011). Nestin and musashi 1 are specific to neural progenitor cells and are not expressed in fully differentiated neurons.

In transplantation of NSCs into the cortex of rats submitted to TBI, $1-3 \%$ of cells became engrafted after 2 weeks of treatment. NSC engraftment and transdifferentiation was associated with an improvement in motor function, but no change in cognitive function was noted (Harting et al., 2009). However, other authors of similar studies have found improved cognitive function in rats (Park et al., 2012).

NSCs systemically infused $2 \mathrm{~h}$ after TBI showed reduction in neurological decline, edema formation, inflammatory infiltration, apoptosis as well as attenuation of activity of Tumor Necrosis Factors alpha (TNF- $\alpha$ ), Interleukin 6 (IL-6) and the transcription factor NF- $\kappa$, compared to other treatment types (Lee et al., 2008). Other authors suggested an optimal time point for transplant of 714 days post injury. At this juncture, the microenvironment of the lesion has low levels of cytokines (TNF, IL-1 $\alpha$, IL-1 $\beta$ and IL-6) where high cytokine levels can have a neurotoxic action (Zhu et al., 2006). Beyond this time window the glial scar forms a barrier enveloping the lesion site which inhibits the local blood circulation vital for graft survival (Bhalala et al., 2012).

The fundamental challenges to successful translation of the large body of pre-clinical work into clinical practice involve the delivery of NSCs to the target location. Direct implantation and intrathecal, intravenous and intra-arterial infusion, has shown low engraftment rates and risk of distal emboli (in intravascular infusions). Novel delivery methods such as nanofiber scaffold implantation could provide the structural and nutritive support required for NSCs proliferation, engraftment and differentiation (Walker et al., 2009).

\subsection{Exogenous Induced Pluripotent Stem Cells (iPS)}

Induced pluripotent cells hold great promise for their potential use in stem cell-based therapy. A number of authors believe the ectopic expression of 4 transcription factors (Oct4, Sox2, Myc and Klf4) was sufficient to reprogram the differentiation status of somatic cells to the pluripotent state of ESCs (Ramos-Mejia et al., 2012). Breton et al. (2013) showed that the transduction of different positions of the pluripotency factors (e.g., Oct4, Sox2, Nanog and LIN28) can have a similar effect on reprogramming of cell lines in equines and humans for pluripotent cells that exhibit the essential features of ESCs. Thus, iPS has the advantage of being derived from the patient thereby precluding the risk of rejection.

Although promising, iPS has the drawbacks of low efficiency of reprogramming, risk of using viral infection in their production and formation of tumors after transplantation. Thus, there are several hurdles to overcome before iPS cells can be considered a potential patient-specific cell therapy (Orlacchio et al., 2010).

\subsection{Exogenous Mesenchymal Stem Cells (MSCs)}

Mesenchymal Stem Cells are multipotent and first isolated from bone marrow. These cells can be administered intravenously or injected directly into the site of the brain lesion for a better outcome (Lam et al., 2013). The action of MSCs involves the secretion of growth factors, exchange of genes and proteins through cell-tocell fusion or contact (Kim et al., 2010), induction of angiogenesis (Khalili et al., 2012) and immunomodulation effects (Sarnowska et al., 2009). The main advantages of using this cell type over other types transplanted cell (e.g., umbilical cord blood, mobilized peripheral blood and neural stem/progenitor cells) is their ease of obtention, potential for 
autologous transplant, fast expansion ex vivo, immune privilege of allogeneic cells and ability to migrate to the site of inflammation (Uccelli et al., 2006).

The factors secreted by MSCs include: (Glial CellLine Derived Neurotrophic Factor (GDNF), BrainDerived Neurotrophic Factor (BDNF), Nerve Growth Factor (NGF), Vascular Endothelial Growth Factor (VEGF), among others (Rooney et al., 2009; Tan et al., 2011; Alder et al., 2012). MSC cultures in supernatant from rat brain submitted to closed traumatic brain trauma also showed increased BDNF, NGF and VEGF, in addition to an increase in Hepatocyte Growth Factor (HGF) (Kim et al., 2010). The large number of factors secreted can promote self-repair of residing tissue cells.

Several pre-clinical trials investigating the use of MSCs in TBI models have shown the migration of cells away from the lesion site and subsequent survival of MSCs, as well as their differentiation into neurons and astrocytes, leading to enhanced motor function (Wang et al., 2012).

The considerable therapeutic potential of human multipotent Mesenchymal Stromal Cells has generated markedly increasing interest from a wide variety of biomedical disciplines. However, investigators report MSCs studies using different methods of isolation and expansion and different approaches to characterizing the cells. Thus, it is increasingly difficult to compare and contrast study outcomes, hindering progress in the field. To begin to address this issue, the Mesenchymal and Tissue Stem Cell Committee of the International Society for Cellular Therapy proposes minimal criteria to define human MSCs. First, MSCs must be plastic-adherent when maintained under standard culture conditions. Second, MSCs must express CD105, CD73 and CD90 and lack expression of CD45, CD34, CD14 or CD11b, CD79a or CD19 and HLA-DR surface molecules. Third, MSCs must differentiate into osteoblasts, adipocytes and chondroblasts in vitro (Vallone et al., 2013).

Several publications have previously demonstrated that the intravenous delivery of MSCs after traumatic brain injury affords neuroprotection via interaction with splenocytes, leading to an increase in systemic antiinflammatory cytokines. (Scheibe et al., 2012; Walker et al., 2010). MSCs have also been shown to differentiate in vivo and in vitro. Markers for human MSCs include: CD10, CD13, CD49b, CD49d, CDw90 and Flk1 (Montesinos et al., 2009; Gong et al., 2011).

The umbilical cord is an abundant source of hematopoietic stem cells. However, investigations reveal a subtype of cells from umbilical cord which do not express marking with CD45 (hematopoietic cell marker) and do not differentiate into hematopoietic cells in vitro. This fraction of mononuclear cells can be expanded in culture and upon stimulation by basic Fibroblast Growth Factor (bFGF) and human epidermal growth factor (hEGF), differentiate into cells which mark positively for the neural markers beta-tubulin III and Glial Fibrillary Acidic Protein (GFAP) (Bicknese et al., 2002).

Mononuclear stromal cells from umbilical cord have a substantial inflammatory, angiogenic effect and have exhibited engraftment rates of over $20 \%$ when transplanted into the Subventricular Zone (SVZ) of neonatal rats, making them attractive therapeutic agents (Walczak et al., 2010).

\subsection{Exogenous Adipose-Derived Stem Cells (ADSCs)}

ADSCs represent a promising source of large quantities of stem cells (Xue et al., 2010) found improvements in motor function (differentiation in neurons and oligodendrocytes) in a spinal cord injury model in rats after intravenous administration of ADSCs (Arboleda et al., 2011).

Another study investigated whether transplantation of Schwann cells differentiated from Adipose-Derived Stem Cells (ADSC-SCs) of rats could promote functional improvement after contusion brain injury, with a focus on effects on reactive gliosis. ADSCs were isolated and expanded from groin adipose tissue of rats and then differentiated into Schwann cells. ADSC-SCs were transplanted into contused rat brain. Immunofluorescence and Western blotting were used to analyse reactive gliosis. In conclusion, transplantation of ADSC-SCs can effectively promote locomotor functional recovery and reduce reactive gliosis after contusion brain injury in rats. ADSCs represent a strong candidate for future research in cell therapies (Yang et al., 2011).

\subsection{Endogenous Stem Cell Therapy}

Endogenous neurogenesis in adults was first described by (Luskin et al., 1997) and later confirmed by other authors (Sawamoto et al., 2011), who showed the presence of Neural Stem Progenitor Cells (NSPCs) within the Subventricular Zona (SVZ) of adult rats that migrated to the olfactory bulb and joined the neural network known as the Rostral Migratory Stream (RMS).

Normal adult brain has two different regions with continuous growth generating new neurons: the SVZ located proximal to the lateral ventricles and the granule cell layer and the Subgranular Zone (SGZ) of the dentate gyrus of the hippocampus (Patzke et al., 2013). Evidence suggests that the adult brain has the potential for compensatory striatal neurogenesis, although the level of 
this neurogenesis is insufficient to counter the progressive neuronal loss which takes place in a diseased or damaged adult brain. Consequently, therapeutic methods may be harnessed to boost neurogenesis and direct migration of progenitor cells towards those specific areas of the brain with neuronal loss.

The studies available indicate an increase in progenitor proliferation of the SVZ region in response to TBI. Other studies however, have found reduced progenitor proliferation in the SVZ region post-TBI, suggesting that the extent of damage in different lesions can result in commensurately different molecular responses both proximal to the lesion and also in the SVZ, giving rise to disparities in cell proliferation within the SVZ (Eriksson et al., 1998).

Adult multipotent precursors are not limited to the olfactory epithelium, anterior SVZ and hippocampus of the adult mammalian brain, having been cultured in vitro from caudal portions of the SVZ, the septum, striatum, cortex, optic nerve, spinal cord and retina. This feature augments the efficacy potential of neuronal replacement therapies based on in situ manipulation of endogenous precursors (Emsley et al., 2004).

Precursors derived from all these regions can selfrenew and differentiate into neurons, astroglia and oligodendroglia in vitro. It is thought that they normally differentiate only into glia or die in vivo. Cells from each region have different requirements for proliferation and differentiation. For instance, precursors derived from septum, striatum, cortex and optic nerve have been reported to require FGF-2 to proliferate and differentiate into neurons in vitro.

Endogenous stimulation occurs mainly due to the release of growth factors such as EGF, FGF-2, bFGF, aFGF, BDNF, NGF, NT-3, VEGF, GDNF, IGF-1 and SDF-1 alpha (Li et al., 2010). These growth factors can be administered by intraventricular, intraparenchymal or intrathecal injection. Some authors report that growth factors, besides improving proliferation, also promote increased migration and gliogenesis of Neural Precursor Cells (NPCs) (Sirko et al., 2010). However, the exact physiopathological mechanisms of functional recovery remain unknown.

Endogenous stimulation has the advantages of avoiding any ethics problems (occurring with embryonic and fetal cells), being less invasive and having no rejection problems (present in exogenous NSPCs) (Saha et al., 2012).

Several authors have described the CBP/p300phosphorylated Smad complex. This CBP/p300phosphorylated Smad complex, when bound to NSCs, can determine their differentiation. If the complex is trapped with Signal Transducers and Activators of Transcription (STAT) phosphorylated 3, the NSCs differentiate into cells with astroglia lineage. Conversely, if the complex is bound with the proneural basic HelixLoop-Helix (bHLH) factor, such as neurogenin 1 and 2, cells differentiate into a neuronal lineage (Herrera et al., 2010). The Sox 2 gene may also play a key role in neural differentiation (Lodato et al., 2013).

Once the NSCs decide to differentiate into neuronal lineage, a cascade of hundreds of genes is regulated throughout neurogenesis to transform the immature neuron into its mature phenotype. Many of these neural genes are controlled by RE1-Silencing Transcription Factor (REST). REST acts as a repressor of neuronal genes in non-neural cells whereas REST regulation activates the large networks of genes required for neural differentiation (Dewald et al., 2011).

Brain-Derived Neurotrophic Factor (BDNF) is also important in regulating the survival and destiny of progenitor cells in adult brain. Studies have been conducted comparing BDNF of healthy versus damaged brain in terms of the distribution of progenitor cells and levels of differentiation and survival. BDNF was overexpressed in the SVZ via recombinant AdenoAssociated Virus (AAV1/2) delivery and newly generated cells were identified using Bromodeoxyuridine (BrdU) labeling. Selective striatal cell loss was induced in a subgroup of rats by unilateral striatal injection of Quinolinic Acid (QA) 21 days after AAV1/2 injection. BDNF has been shown to promote both neuronal differentiation and the survival of newly generated daughter cells (Henry et al., 2007; Kells and Connor, 2008). In vivo, BDNF delivery to SVZ-derived adult neural progenitor cells increased neuronal recruitment to the olfactory bulb (Tanaka et al., 2010) and resulted in ectopic addition of newly generated neurons, expressing markers of $\gamma$-aminobutyric acid (GABA) ergic medium spiny striatal neurons, to the striatum of normal adult rat brain (Hou et al., 2008). This evidence points to the conclusion that elevated levels of BDNF, induced by injury, can increase the recruitment of progenitor cells to the site of lesioned striatum and promote neuronal differentiation in both normal and damaged striatum. Increasing BDNF expression may be a viable strategy for augmenting neurogenesis of endogenous progenitor cells (Chen et al., 2012).

A large cell-surface carbohydrate, Polysialic Acid (PSA), regulates cell interactions and is harnessed during vertebrate development to promote precursor cell migration and axon path-finding. The induction of PSA expression in damaged adult CNS tissues could help them rebuild by creating conducive conditions for architectural remodeling. This possibility was explored 
through two approaches: the regeneration of axons; and the recruitment of endogenous neural precursors to a lesion site. Glial scars that form at CNS injury sites block axon regeneration. It was found that transfection of scar astrocytes by a viral vector encoding polysialyltransferase leads to sustained expression of high levels of PSA. With this treatment, a substantial proportion of severed corticospinal tract axon processes were able to grow through a spinal injury site. Studies of precursor cell migration to a cortical lesion have found that induced PSA expression, in a path extending from the subventricular zone to a lesion near the cortical surface, increased recruitment of BrdU/nestin-positive cells along the path and into the injury site. The displaced precursors were able to differentiate in a regionally appropriate manner. These results suggest that induced PSA expression can be used as a strategy for promoting tissue repair involving both replacement of cells and rebuilding of neural connections (Luo et al., 2011).

\subsection{Therapeutic Mechanism-the Three Hypotese}

While initial research has indicated that engraftment and transdifferentiation into neural cells could explain the observed benefit, the exact underlying mechanism of improvement remains controversial. A second hypothesis implicates localized stem/progenitor cell engraftment with subsequent alteration of the loco-regional milieu; however, the limited rate of cell engraftment makes this theory less plausible. There is a growing body of preclinical data supporting the notion that, after intravenous injection, stem/progenitor cells interact with immunologic cells located in organ systems distal to the CNS, thereby altering the systemic immunologic/inflammatory response. Such remote cell "bioreactors" may modulate the observed post-injury pro-inflammatory environment and lead to neuroprotection (Walker et al., 2011).

\subsection{Engraftment and Transdifferentiation}

Lundberg et al. (2009) administered human MSCs into the ipsilateral carotid artery of rats submitted to TBI. The intra-arterial transplantation of MSCs resulted in migration to the lesion and engraftment of the CNS without thromboembolic ischemia.

The Ha laboratory implanted Human Umbilical Cord Blood Mononuclear Cells (HUCBCs) into the injured region and found engrafted HUCBCs up to 8 week after injury. HUCBCs were found to express the neural markers GFAP and Microtubule-Associated Protein 2 (MAP2).
Functional improvement via locomotor testing was observed in the animals for up to 8 week (CalatravaFerreras et al., 2012).

The concept of plasticity of stem cells or transdifferentiation could explain the ability of stem cells of one adult tissue to proliferate into different cell lineages (i.e., engraftment of neurons after MSCs therapy) (Li and Chopp, 2009).

The differentiation of MSCs into neural cell lineages in vitro uses compounds such as $\beta$-mercaptoethanol (Nichols et al., 2013), Dimethyl Sulfoxide (DMSO) (Barnabe et al., 2009), retinoic acid (Jiang et al., 2012) and growth factors FGF and EGF (Aizman et al., 2013). The forming of neurospheres has also been observed, where these are able to produce neurons or astrocytes and oligodendrocytes, visualized by specific immunohistochemical markers (Birenboim et al., 2013).

Although promising studies have shown engraftment and transdifferentiation of progenitor cells transplanted into neural tissue, the importance of engraftment and frequency of transdifferentiation remain extremely controversial in the literature (Zhang and Alexanian 2012), others claim that neural markers may be merely evidencing the result of extreme cellular stress and artifacts of the technique, particularly after the use of aggressive substances such as beta-mercaptoethanol and DMSO (Lu et al., 2004).

\subsection{Modulation of the Locoregional Inflammatory Milieu}

Progenitor cell migration towards the site of injury and interaction with resident microglia could modulate the locoregional inflammatory response thereby leading to enhanced neuroprotection. The recruitment of inflammatory cells is known to lead to the cascade of secondary damage common to injuries of the Central Nervous System (CNS). Cell activation and infiltration into the injury site is measured by changes in the expression of chemokines (IL-1 alpha, IL-1 beta, IL-6 and TNF alpha), the chemoattractive cytokines. Cocultures of MSCs with purified immune natural killerlike cells, dendritic cells and both naïve and effector $\mathrm{T}$ cells, increase production of anti-inflammatory interleukins IL-4 and IL-10, while reducing the quantity of TNF- $\alpha$ and IFN- $\gamma$. Increase in IL- 4 together with reduction in IFN- $\gamma$ promotes a change in the subset of $\mathrm{T}$ helper cells, from Th1 cytotoxic cells to Th2 cells. In addition, reduction in TNF- $\alpha$ allied with increase in IL10 can reduce the maturation of dendritic cells, while increasing the number of regulatory the $\mathrm{T}$ cells that 
promote an anti-inflammatory or tolerant response (Walker et al., 2010).

Reducing the number of inflammatory cells requested by blocking the action of chemokines has proved a promising approach for reducing neuroinflammation and improving tissue preservation and neovascularization. In addition, several chemokines have been shown to be essential for stem/progenitor cell attraction, survival, differentiation and cytokine production. Thus, chemokines may be indirectly involved in remyelination, revascularization and neuroprotection, important prerequisites for CNS repair after trauma (Jaerve and Mueller, 2012).

Walker et al. (2010) implanted MSCs directly into the cortex of rats with TBI and found increased Interleukin 6 (IL-6) in brain tissue supernatant. Subsequently, a series of MSCs in vitro and co-cultures of NSCs showed activation of the NSC NFkB pathway leading to a reduction in cellular apoptosis.

In situations of brain ischemia, trauma or various other pathologies, astrocytes are activated causing a phenomenon called reactive astrogliosis. This process is characterized by hypertrophy, cell proliferation, extension of cell processes, as well as increase in the production of the proteins GFAP, vimentin and nestin (Weber et al., 2013).

Reactive gliosis results in the formation of glial scars. Currently, neural scarring is believed to be responsible for the growth inhibition of neurites, hampering regeneration in the CNS after lesions. In addition, this scarring inhibits communication among existing neuronal processes (Yu et al., 2012).

\subsection{Modulation of the Systemic Immunologic Response}

Stem cell therapy influences the systemic immunologic response, triggering an increase in antiinflammatory cytokine and the production of regulatory T-cells, with decreased production of cytotoxic T-cells (Ying et al., 2011). Recently, a study in a rat model of stroke showed that, following stroke, spleen size was reduced concomitantly with CD8+ T-cell counts. The transfusion of systemic HUCBCs in a rat model of stroke showed recovery of spleen weight, splenic CD8+ T-cell counts, as well as extent of brain injury. Moreover, the proliferation of T-cells was reduced commensurate with the increase in IL-10 and a reduction in IFN- $\gamma$. The reduced peripheral inflammatory response was associated with a reduction in the volume of the brain infarct by up to $85 \%$ (Vendrame et al., 2005).
Theories have also been proposed suggesting that stem cell treatment induces angiogenesis, predominantly responsible for tissue repair (Xiong et al., 2012). In a rat model, intravenous injection of MSCs after induced stroke resulted in augmented levels of endogenous VEGF of the VEGF 2 receptor, as well as increased angiogenesis in the transition zone (Caplan and Dennis, 2006).

It is important to point out that the immune response in the brain is limited and peculiar, a feature afforded by the selective permeability of the blood-brain barrier. However, this is known to be only partially true since in certain situations, infiltration by other cells, especially lymphocytes, can be seen (Sekeljic et al., 2012). In the majority of cases, only the microglia and astrocytes are activated, where these cell types, particularly the former, are responsible for the immunological response in the CNS (Ikeshima-Kataoka et al., 2013).

\section{CONCLUSION}

Mortality and morbidity of TBI are set to increase globally in coming years. The preclinical work reviewed in this study offers novel data supporting the potential efficacy of cell therapies for TBI. It is now possible to accompany autologous neural stem cells in vivo and cell migration, confirming that neural stem cells can selectively target brain injuries and undergo neurogenesis. However, this field is only beginning to understand the complex interplay between neural precursor potential and signalling in their local microenvironment. Although neurogenesis normally occurs in only two areas of the adult brain (SVZ and dentate gyrus), other research suggests that it may be possible to manipulate endogenous neural precursors in situ to undergo neurogenesis in other regions of the adult brain. Multipotent precursors capable of differentiating into neurons, astroglia and oligodendroglia exist in many regions of the adult brain. These precursors have considerable plasticity and despite limitations integrating into some areas of the CNS, appear capable of differentiating into neurons appropriate for a wide variety of regions when heterotopically transplanted, or, more recently, recruited in situ.

Looking forward, additional preclinical research is warranted to further elucidate the progenitor cell mechanism of action and to aid the planning of quality controlled clinical studies. This holds the key to advancing in the treatment of TBI using stem cell therapy.

\subsection{Conflict of Interests}

The authors declare that they have no conflict of interest. 


\section{ACKNOWLEDGMENT}

The researchers would like to thank Capes Brazil for the excellence in research scholarship.

\section{REFERENCES}

Abdullah, A.I., A. Pollock and T. Sun, 2012. The path from skin to brain: Generation of functional neurons from fibroblasts. Mol. Neurobiol., 45: 586-595. DOI: $10.1007 / \mathrm{s} 12035-012-8277-6$

Aizman, I., M. McGrogan and C.C. Case, 2013. Quantitative microplate assay for studying mesenchymal stromal cell-induced neuropoiesis. Stem Cells Transl. Med., 2: 223-232. DOI: 10.5966/sctm.2012-0119

Albert-Weibenberger, C., C. Varrallyay, F. Raslan, C. Kleinschnitz and A.L. Siren, 2012. An experimental protocol for mimicking pathomechanisms of traumatic brain injury in mice. Exp. Transl Stroke Med., 4: 1-11. DOI: 10.1186/2040-7378-4-1

Alder, J., B.C. Kramer, C. Hoskin and S. Thakker-Varia, 2012. Brain-derived neurotrophic factor produced by human umbilical tissue-derived cells is required for its effect on hippocampal dendritic differentiation. Dev. Neurobiol., 72: 755-765. DOI: 10.1002/dneu.20980

Arboleda, D., S. Forostyak, P. Jendelova, D. Marekova and T. Amemori et al., 2011. Transplantation of predifferentiated adipose-derived stromal cells for the treatment of spinal cord injury. Cell Mol. Neurobiol., 31: 113-122 DOI: 10.1007/s10571-0119712-3

Archer, T.C., J. Jin and E.S. Casey, 2011. Interaction of Sox1, Sox2, Sox3 and Oct4 during primary neurogenesis. Dev. Biol., 2: 429-440. DOI: 10.1016/j.ydbio.2010.12.013

Barnabe, G.F., T.T. Schwindt, M.E. Calcagnotto, F.L. Motta and G. Jr. Martinez et al., 2009. Chemicallyinduced RAT mesenchymal stem cells adopt molecular properties of neuronal-like cells but do not have basic neuronal functional properties. PLoS One, 4: e5222-e5222. DOI: 10.1371/journal.pone.0005222

Bhalala, O.G., L. Pan, V. Sahni, T.L. McGuire and K. Gruner, et al., 2012. MicroRNA-21 regulates astrocytic response following spinal cord injury. J. Neurosci., 32: 17935-17947. DOI: 10.1523/ JNEUROSCI.3860-12.2012

Bicknese, A.R., H.S. Goodwin, C.O. Quinn, V.C. Henderson and S.N. Chien, 2002. Human umbilical cord blood cells can be induced to express markers for neurons and glia. Cell Transplant., 11: 261-264. PMID: 12075991
Birenboim, R., A. Markus and R.S. Goldstein, 2013. Simple generation of neurons from human embryonic stem cells using agarose multiwell dishes. J. Neurosci. Methods, 214: 9-14. DOI: 10.1016/j.jneumeth.2012.12.026

Breton, A., R. Sharma, A.C. Diaz, A.G. Parham and A. Graham et al., 2013. Derivation and characterization of induced pluripotent stem cells from equine fibroblasts. Stem Cells Dev., 22: 611-621. DOI: $10.1089 / \mathrm{scd} .2012 .0052$

Calatrava-Ferreras, L., R. Gonzalo-Gobernado, A.S. Herranz, D. Reimers and T. Montero Vega et al., 2012. Effects of intravenous administration of human umbilical cord blood stem cells in 3acetylpyridine-lesioned rats. Stem Cells Int., 2012: 135187-135187. DOI: 10.1155/2012/135187

Caplan, A.I. and J.E. Dennis, 2006. Mesenchymal stem cells as trophic mediators. J. Cell Biochem., 5: 1076-1084. DOI: $10.1002 /$ jcb.20886

Chen, S.J., J.C. Tsai, C.Y. Lin, C.K. Chang and T.H. Tseng et al., 2012. Brain-derived neurotrophic factor-transfected and nontransfected $3 \mathrm{t} 3$ fibroblasts enhance migratory neuroblasts and functional restoration in mice with intracerebral hemorrhage. $\mathrm{J}$. Neuropathol. Exp. Neurol., 71: 1123-1136. DOI: 10.1097/NEN.0b013e3182779e96

Cheng, G., R.H. Kong, L.M. Zhang and J.N. Zhang, 2012. Mitochondria in traumatic brain injury and mitochondrial-targeted multipotential therapeutic strategies. Br. J. Pharmacol., 167: 699-719. DOI: 10.1111/j.1476-5381.2012.02025.x

Cristini, S., G. Alessandri, F. Acerbi, E. Ciusani and A. Colombo et al., 2011. Three-dimensional selforganizing neural architectures: A neural stem cells reservoir and a system for neurodevelopmental studies. Tissue Eng. Part. C. Methods, 17: 11091120. DOI: 10.1089/ten.TEC.2010.0622

Dardiotis, E., S. Grigoriadis and G.M. Hadjigeorgiou, 2012. Genetic factors influencing outcome from neurotrauma. Curr. Opin. Psychiatry, 25: 231-238. DOI: $10.1097 /$ YCO.0b013e3283523c0e

Dewald, L.E., J.P. Rodriguez and J.M. Levine, 2011. The RE1 binding protein REST regulates oligodendrocyte differentiation. J. Neurosci., 31: 3470-3483. DOI: 10.1523/JNEUROSCI.276810.2011

Emsley, J.G., B.D. Michell, S.S. Magavi, P. Ariotta and J.D. Macklis, 2004. The repair of complex neuronal circuitry by transplanted and endogenous precursors. NeuroRx., 1: 452-471. PMID: 15717047 
Erceg, S.M.R. and M. Stojkovic, 2009. Human embryonic stem cell differentiation toward regional specific neural precursors. Stem Cells, 1: 78-87. DOI: 10.1634/stemcells.2008-0543

Eriksson, P.S., E. Perfilieva, T. Bjork-Eriksson, A.M. Alborn and C. Nordborg et al., 1998. Neurogenesis in the adult human hippocampus. Nat. Med., 4: 1313-1317. DOI: $10.1038 / 3305$

Foti, S.B., A. Chou, A.D. Moll and A.J. Roskams, 2013. HDAC inhibitors dysregulate neural stem cell activity in the postnatal mouse brain. Int. J. Dev. Neurosci. DOI: 10.1016/j.ijdevneu.2013.03.008

Gong, M., Y. Bi, W. Jiang, Y. Zhang and L. Chen et al., 2011. Immortalized mesenchymal stem cells: An alternative to primary mesenchymal stem cells in neuronal differentiation and neuroregeneration associated studies. J. Biomed. Sci., 18: 87-87. DOI: 10.1186/1423-0127-18-87

Harting, M.T., L.E. Sloan, F. Jimenez, J. Baumgartner and C.S.J. Cox, 2009. Subacute neural stem cell therapy for traumatic brain injury. J. Surg Res., 15: 188-194. DOI: $10.1016 /$ j.jss.2008.03.037

Heile, A. and T. Brinker, 2011. Clinical translation of stem cell therapy in traumatic brain injury: The potential of encapsulated mesenchymal cell biodelivery of glucagon-like peptide-1. Dialogues Clin. Neurosci., 13: 279-286. PMID: 22034462

Henry, R.A., S.M. Hughes and B. Connor, 2007. AAVmediated delivery of BDNF augments neurogenesis in the normal and quinolinic acid-lesioned adult rat brain. Eur. J. Neurosci., 25: 3513-3525. DOI: 10.1111/j.1460-9568.2007.05625.x

Herrera, F., Q. Chen and D. Schubert, 2010. Synergistic effect of retinoic acid and cytokines on the regulation of glial fibrillary acidic protein expression. J. Biol. Chem., 285: 38915-38922. DOI: 10.1074/jbc.M110.170274

Hinzman, J.M., T.C. Thomas, J.E. Quintero, G.A Gerhardt and J. Lifshitz, 2012. Disruptions in the regulation of extracellular glutamate by neurons and glia in the rat striatum two days after diffuse brain injury. J. Neurotrauma, 10: 1197-1208. DOI: 10.1089/neu.2011.2261

Hou, S.W., Y.Q. Wang, M. Xu, D.H. Shen and J.J. Wang et al., 2008. Functional integration of newly generated neurons into striatum after cerebral ischemia in the adult rat brain. Stroke, 39: 2837-2844.

Ikeshima-Kataoka, H., Y. Abe, T. Abe and M. Yasui, 2013. Immunological function of aquaporin-4 in stab-wounded mouse brain in concert with a proinflammatory cytokine inducer, osteopontin. Mol. Cell Neurosci. DOI: 10.1016/j.mcn.2013.02.002
Jaerve, A. and H.W. Muller, 2012. Chemokines in CNS injury and repair. Cell Tissue Res., 349: 229-248. DOI: $10.1007 / \mathrm{s} 00441-012-1427-3$

Jiang, X., H.Q. Cao, L.Y. Shi, S.Y. Ng and L.W. Stanton et al., 2012. Nanofiber topography and sustained biochemical signaling enhance human mesenchymal stem cell neural commitment. Acta Biomater, 8: 1290-1302. DOI: 10.1016/j.actbio.2011.11.019

Kahle, K.T., B.P. Walcott and J.M. Simard, 2013. Continuous hyperosmolar therapy for traumatic brain injury-associated cerebral edema: As good as it gets, or an iatrogenic secondary insult? J. Clin. Neurosci., 20 : 30-41. DOI: 10.1016/j.jocn.2012.10.004

Kells, A.P and B. Connor, 2008. AAV-mediated expression of $\mathrm{Bcl}-\mathrm{xL}$ or XIAP fails to induce neuronal resistance against quinolinic acid-induced striatal lesioning. Neurosci. Lett., 436: 326-330. DOI: 10.1016/j.neulet.2008.03.051

Kelso, M.L. and J.H. Oestreich, 2012. Traumatic brain injury: Central and peripheral role of $\alpha 7$ nicotinic acetylcholine receptors. Curr. Drug Targets, 13: 631-636. PMID: 22300031

Khalili, M.A., M. Anvari, S.H, Hekmati-Moghadam, F. Sadeghian-Nodoushan and F. Fesahat et al., 2012. Therapeutic benefit of intravenous transplantation of mesenchymal stem cells after experimental subarachnoid hemorrhage in rats. J Stroke Cerebrovasc Dis., 6: 445-451. DOI: 10.1016/j.jstrokecerebrovasdis.2010.10.005

Kim, H.J., J.H. Lee and S.H. Kim, 2010. Therapeutic effects of human mesenchymal stem cells on traumatic brain injury in rats: Secretion of neurotrophic factors and inhibition of apoptosis. J. Neurotrauma, 27: $131-138 . \quad$ DOI: 10.1089/NEU.2008-0818

Kumar, A. and D.J. Loane, 2012. Neuroinflammation after traumatic brain injury: Opportunities for therapeutic intervention. Brain Behav. Immun., 26: 1191-1201. DOI: 10.1016/j.bbi.2012.06.008

Lam, P.K., A.W. Lo, K.K. Wang, H.C. Lau and K.T. Li et al., 2013. Transplantation of mesenchymal stem cells to the brain by topical application in an experimental traumatic brain injury model. J. Clin. Neurosci., $20: \quad 306-309 . \quad$ DOI: 10.1016/j.jocn.2012.03.028

Leao, R.N., A. Reis, A. Emirandetti, M. Lewicka and O. Hermanson, 2010. A voltage-sensitive dye-based assay for the identification of differentiated neurons derived from embryonic neural stem cell cultures. PLoS. One, 5: e13833-e13833. DOI: 10.1371/journal.pone.0013833 
Lee, S.T., K. Chu, K.H. Jung, S.J. Kim and D.H. Kim et al., 2008. Anti-inflammatory mechanism of intravascular neural stem cell transplantation in haemorrhagic stroke. Brain, 131: 616-629. DOI: 10.1093/brain/awm306

Li, Y. and M. Chopp, 2009. Marrow stromal cell transplantation in stroke and traumatic brain injury. Neurosci. Lett., 456: 120-123. DOI: 10.1016/j.neulet.2008.03.096

Li, Y., G. Calfa, T. Inoue, M.D. Amaral and L. PozzoMiller, 2010. Activity-dependent release of endogenous bdnf from mossy fibers evokes a trpc3 current and $\mathrm{Ca} 2+$ elevations in $\mathrm{Ca} 3$ pyramidal neurons. J. Neurophysiol., 103: 2846-2856. DOI: 10.1152/jn.01140.2009

Lodato, M.A., C.W. Ng, J.A. Wamstad, A.W. Cheng and K.K. Thai et al., 2013. SOX2 Co-Occupies distal enhancer elements with distinct POU factors in ESCs and NPCs to specify cell state. PLoS. Genet., 9: e1003288-e1003288. DOI: 10.1371/journal.pgen. 1003288

Lok, J., W. Leung and S. Zhao, 2011. Gammaglutamylcysteine ethyl ester protects cerebral endothelial cells during injury and decreases bloodbrain barrier permeability after experimental brain trauma. J. Neurochem., 118: 248-255. DOI: 10.1111/j.1471-4159.2011.07294.x

Lu, P., A. Blesch and M.H. Tuszynski, 2004. Induction of bone marrow stromal cells to neurons: Differentiation, transdifferentiation, or artifact? J. Neurosci. Res., 77: 174-191. DOI: 10.1002/jnr.20148

Lundberg, J., K. Le Blanc, M. Soderman, T. Andersson and S. Holmin, 2009. Endovascular transplantation of stem cells to the injured rat CNS. Neuroradiology, 10: 661-667. DOI: 10.1007/s00234-009-0551-6

Luo, J., X. Bo, D. Wu, J. Yeh and P.M. Richardson et al., 2011. Promoting survival, migration and integration of transplanted Schwann cells by overexpressing polysialic acid. Glia, 59: 424-434. DOI: 10.1002/glia.21111

Luskin, M.B., T. Zigova, B.J. Soteres and R.R. Stewart, 1997. Neuronal Progenitor cells derived from the anterior subventricular zone of the neonatal rat forebrain continue to proliferate in vitro and express a neuronal phenotype. Mol. Cell Neurosci., 8: 351366. DOI: 10.1006/mcne.1996.0592

Montesinos, J.J., E. Flores-Figueroa, S. Castillo-Medina, P. Flores-Guzman and E. Hermandez-Estevez et al., 2009. Human mesenchymal stromal cells from adult and neonatal sources: comparative analysis of their morphology, immunophenotype, differentiation patterns and neural protein expression. Oncol. Res., 11: 163-176. DOI: 10.1080/14653240802582075
Nichols, J.E., J.A. Niles, D. Dewitt, D Prough and M. Parsley et al., 2013. Neurogenic and neuroprotective potential of a novel subpopulation of peripheral blood-derived CD133+ ABCG2+CXCR4+ mesenchymal stem cells: Development of autologous cell-based therapeutics for traumatic brain injury. Stem Cell Res. Ther., 6: 3-3. PMID: 23290300

Oliva, A.A., Y. Kang, J. Sanchez, J.M. Hinzman and T.C. Thomas et al., 2012. Disruptions in the regulation of extracellular glutamate by neurons and glia in the rat striatum two days after diffuse brain injury. J. Neurotrauma, 29: 1197-1208. DOI: 10.1089/neu.2011.2261

Orlacchio, A., G. Bernardi, A. Orlacchio and S. Martino, 2010. Stem cells: An overview of the current status of therapies for central and peripheral nervous system diseases. Curr. Med. Chem., 17: 595-608. PMID: 20088765

Park, D., S.S. Joo, T.K. Kim, S.H. Lee and H. Kang et al., 2012. Human neural stem cells overexpressing choline acetyltransferase restore cognitive function of kainic acid-induced learning and memory deficit animals. Cell Transplant., 21: 365-371. DOI: $10.3727 / 096368911$ X586765

Patzke, N., C. Kaswera, E. Gilissen, A.O. Ihunwo and P.R. Manger, 2013. Adult neurogenesis in a giant otter shrew (Potamogale velox). Neuroscience. DOI: 10.1016/j.neuroscience.2013.02.025

Qu, X., T. Liu, K. Song, X. Li and D. Ge, 2012. Induced pluripotent stem cells generated from human adipose-derived stem cells using a non-viral polycistronic plasmid in feeder-free conditions. PLoS. One, 7: e48161-e48161. DOI: 10.1371/journal.pone.0048161

Ramos-Mejia, V., R. Montes, C. Bueno, V. Ayllon and P.J. Real et al., 2012. Residual expression of the reprogramming factors prevents differentiation of iPSC generated from human fibroblasts and cord blood CD34+ progenitors. PLoS. One, 7: e358247e35824. DOI: 10.1371/journal.pone.0035824

Reis, A and O. Hermanson, 2012. The DNA glycosylases OGG1 and NEIL3 influence differentiation potential, proliferation and senescence-associated signs in neural stem cells. Biochem. Biophys. Res. Commun., 13: 621-626. DOI: 10.1016/j.bbrc.2012.04.125

Rooney, G.E., S.S. Mcmahon, T. Ritter, Y. Garcia and C. Moran et al., 2009. Neurotrophic factor-expressing mesenchymal stem cells survive transplantation into the contused spinal cord without differentiating into neural cells. Tissue Eng. Part. A., 15: 3049-3059. DOI: $10.1089 /$ ten.TEA.2009.0045 
Saha, B., M. Jaber and A. Gaillard, 2012. Potentials of endogenous neural stem cells in cortical repair. Front Cell Neurosci., 6: 14-14. DOI: 10.3389/fncel.2012.00014

Sarnowska, A., H. Braun, S. Sauerzweig and K.G. Reymann, 2009. The neuroprotective effect of bone marrow stem cells is not dependent on direct cell contact with hypoxic injured tissue. Exp. Neurol., 215: 317-327. DOI: 10.1016/j.expneurol.2008.10.023

Sawamoto, K., Y. Hirota, C. Alfaro-Cervello, M. Soriano-Navarro and X. He et al., 2011. Cellular composition and organization of the subventricular zone and rostral migratory stream in the adult and neonatal common marmoset brain. J. Comp. Neurol., 519: 690-713. DOI: 10.1002/cne.22543

Scheibe, F., J. Ladhoff, J. Huck, M. Grohmann and K. Blazej et al., 2012. Immune effects of mesenchymal stromal cells in experimental stroke. J. Cereb. Blood Flow Metab., 32: 1578-1588. PMID: 22549620

Sekeljic, V., D. Bataveljic, S. Stamenkovic, M. Ulamek and M. Jablonski et al., 2012. Cellular markers of neuroinflammation and neurogenesis after ischemic brain injury in the long-term survival rat model. Brain Struct. Funct., 217: 411-420. DOI: 10.1007/s00429-011-0336-7

Sirko, S., A. Holst, A. Weber, A. Wizenmann and U. Theochatidis et al., 2010. Chondroitin sulfates are required for fibroblast growth factor-2-dependent proliferation and maintenance in neural stem cells and for epidermal growth factor-dependent migration of their progeny. Stem Cells, 28: 775-787. DOI: $10.1002 /$ stem.309

Tan, B., Z. Luan, X. Wei, Y. He and G. Wei et al., 2011. AMP-activated kinase mediates adipose stem cellstimulated neuritogenesis of PC12 cells. Neuroscience, 181 : 40-47. DOI: 10.1016/j.neuroscience.2011.02.038

Tanaka, Y., R. Tanaka, M. Liu, N. Hattori and T. Urabe, 2010. Cilostazol attenuates ischemic brain injury and enhances neurogenesis in the subventricular zone of adult mice after transient focal cerebral ischemia. Neuroscience, 171: 1367-1376. DOI: 10.1016/j.neuroscience.2010.10.008

Uccelli, A., L. Moretta and V. Pistoia, 2006. Immunoregulatory function of mesenchymal stem cells. Eur. J. Immunol., 10: 2566-2573. DOI: 10.1002/eji.200636416

Vallone, V.B.F., M.A. Romaniuk, H. Choi, V. Labovsky and J. Otaequi et al., 2013. Mesenchymal stem cells and their use in therapy: What has been achieved? Differentiation, 85 : 1-10. DOI: 10.1016/j.diff.2012.08.004
Vendrame, M., C. Gemma, D. de Mesquita, L. Collier and P.C. Bickford et al., 2005. Anti-inflammatory effects of human cord blood cells in a rat model of stroke. Stem Cells Dev., 14: 595-604. DOI: 10.1089/scd.2005.14.595

Walczak, P., N. Chen, J.E. Hudson, A.E. Willing and S.N. Garbuzova-Davis et al., 2010. Functional endothelial progenitor cells derived from adipose tissue show beneficial effect on cell therapy of traumatic brain injury. Neurosci. Lett., 473: 186191. DOI: 10.1016/j.neulet.2010.02.035

Walker, P.A., K.R. Aroom, F. Jimenez, S.K. Shah and M.T. Harting, 2009. Advances in progenitor cell therapy using scaffolding constructs for central nervous system injury. Stem Cell Rev., 5: 283-300. DOI: $10.1007 / \mathrm{s} 12015-009-9081-1$

Walker, P.A., M.T. Harting, F. Jimenez, S.K. Shah and S. Pati et al., 2010. direct intrathecal implantation of mesenchymal stromal cells leads to enhanced neuroprotection via an NFKB-mediated increase in interleukin-6 production. Stem Cells Dev., 19: 867876. DOI: $10.1089 / \mathrm{scd} .2009 .0188$

Walker, P.A., P.A. Letourneau, S. Bedi, S.K. Shah and F. Jimenez et al., 2011. Progenitor cells as remote "bioreactors": Neuroprotection via modulation of the systemic inflammatory response. World J. Stem Cells, 3: 9-18. DOI: 10.4252/wjsc.v3.i2.9

Wang, S., Q. Kan, Y. Sun, R. Han and G. Zhang et al., 2012. Caveolin-1 regulates neural differentiation of rat bone mesenchymal stem cells into neurons by modulating notch signaling. Int. J. Dev. Neurosci., 31: 30-35. DOI: 10.1016/j.ijdevneu.2012.09.004

Weber, M., N. Scherf, T. Kahl, U.D. Braumann and P. Sheibe et al., 2013. Quantitative analysis of astrogliosis in drug-dependent humans. Brain Res., 1500: 72-87. DOI: 10.1016/j.brainres.2012.12.048

Woodcock, T. and M.C. Morganti-Kossmann, 2013. The role of markers of inflammation in traumatic brain injury. Front. Neurol., 4: 18-18. DOI: 10.3389/fneur.2013.00018

Xiong, Y., A. Mahmood, Y. Meng, Y. Zhang and Z.G. Zhang et al., 2012. Neuroprotective and neurorestorative effects of thymosin $\beta 4$ treatment following experimental traumatic brain injury. Ann. N. Y. Acad. Sci., 1270: 51-58. DOI: 10.1111/j.17496632.2012.06683.x

Xiong, Y., I.N. Singh and E.D. Hall, 2009. Tempol protection of spinal cord mitochondria from peroxynitrite-induced oxidative damage. Free Radic. Res., 43: 604-612. DOI: $10.1080 / 10715760902977432$ 
Xue, S., H.T. Zhang, P. Zhang, J. Luo and Z.Z. Chen et al., 2010. Functional endothelial progenitor cells derived from adipose tissue show beneficial effect on cell therapy of traumatic brain injury. Neurosci. Lett., $\quad 473$ : $186-191 . \quad$ DOI: 10.1016/j.neulet.2010.02.035

Yang, H., G.D. Feng, Z. Liang, A. Vitale and X.Y. Jiao et al., 2012. In vitro beneficial activation of microglial cells by mechanically-injured astrocytes enhances the synthesis and secretion of BDNF through p38MAPK. Neurochem. Int., 61: 175-186. DOI: 10.1016/j.neuint.2012.04.020

Yang, L., J.S. Fang, W. Wang, R.K. Chen and C.F. Shen, 2011. Transplantation of Schwann cells differentiated from adipose-derived stem cells modifies reactive gliosis after contusion brain injury in rats. J. Int. Med. Res., 39: 1344-1357. DOI: 10.1177/147323001103900421

Ying, L., Z. Fu, J. Lu, C. Zhou and Y Chen et al., 2011. Cytotoxic $\mathrm{T}$ lymphocyte antigen 4 immunoglobulin modified dendritic cells attenuate allergic airway inflammation and hyperresponsiveness by regulating the development of T helper type 1 (Th1)/Th2 and $\mathrm{Th} 2 /$ regulatory $\mathrm{T}$ cell subsets in a murine model of asthm. Clin. Exp. Immunol., 165: 130-139. DOI: 10.1111/j.1365-2249.2011.04405.x
Yu, P., H. Wang, Y. Katagiri and H.M. Geller, 2012. An in vitro model of reactive astrogliosis and its effect on neuronal growth. Methods Mol. Biol., 814: 327340. DOI: 10.1007/978-1-61779-452-0_21

Zhang, Z and A.R. Alexanian, 2012. The neural plasticity of early-passage human bone marrowderived mesenchymal stem cells and their modulation with chromatin-modifying agents. J. Tissue Eng. Regen. Med. DOI: 10.1002/term.1535

Zhu, Y., J. Antony, S. Liu, J.A. Martinez and F. Giuliani, 2006. CD8+ lymphocyte-mediated injury of dorsal root ganglion neurons during lentivirus infection: CD154-dependent cell contact neurotoxicity. J. Neurosci., 13: 3396-3403. DOI: 10.1523/JNEUROSCI.4767-05.2006 